Brit. J. vener. Dis. (1956) 32, 229.

\title{
ORGANIZATION OF SYPHILIS CONTROL IN THE SOVIET UNION*†
}

\author{
BY \\ PYOTR VASILIEVICH KOZHEWNIKOV \\ Institute of Advanced Medical Studies for Physicians, Leningrad, U.S.S.R.
}

In Russia there was no effective or planned control of syphilis before 1917 , in spite of the fact that this infection was very widespread in the country. A special congress of Russian syphilologists convened in Petersburg in 1897 outlined all necessary measures in the field of syphilis control but, although the programme suggested by the congress was a very modest one, it was not implemented. After 1917 planned control of venereal diseases was started in the Soviet Union on a state-wide scale. During the first years of the Soviet régime, great attention was paid to the so-called "expedition" method of examining the population to register cases of syphilis, organize prophylaxis and check the spread of venereal diseases. More than 1,000 expeditions and teams were sent to the outlying regions before 1940 .

New forms of dispensary work were found to control venereal diseases, the main principles of which were the following:

(1) Planned character of all measures;

(2) Olbigatory registration of syphilis cases;

(3) Treatment and prophylactic examination free of charge;

(4) Follow-up of patients until complete recovery;

(5) Active epidemiological work;

(6) Prophylaxis;

(7) Health education;

(8) Legal penalties for intentional contamination.

Some brief explanations are necessary in order to understand the above principles of work. Contagious syphilis cases have to be hospitalized within 24 hours. Such cases remain in hospital for a month-and-a-half for the first course of treatment after which treatment is administered at outpatient clinics. The examination, treatment, and follow-up of patients take several years and are free of charge. The physician has to see to it that patients visit the out-patient clinic regularly. Should the patient not visit the clinic at the appointed time, his physician is obliged to summon him (by telephone, letter, or through a specially sent nurse).

\footnotetext{
* Received for publication May 31, 1956.
}

+ Read at First International Symposium on Venereal Diseases and the Treponematoses, Washington, D.C., May 28, 1956.
On diagnosing a new syphilis case, the doctors have to find the source of infection and examine the patient's contacts to find out whether there are any cases of syphilis among them. Some patients in clinics and hospitals, as well as all pregnant women, are examined by serologic methods. All doctors actively participate in the work of syphilis control, each in his own field. Annual reports are presented by districts, regions, and republics. The analysis of what has been done in previous years serves as a basis for outlining the plan for syphilis control for the coming year.

When planning methods of treating syphilis, Soviet scientists find ways of adjusting the treatment to the needs of the individual patient. Thus, for example, taking into account the fact that in a village it is difficult for the patient to visit the clinic several times a week for regular injections of neosalvarsan or bismuth, our venereologists worked out the so-called "one-stage" method of treatment whereby neosalvarsan and bismuth in large doses are administered together. Thus, in spite of the fact that the patient visits the clinic only once in 5 or 6 days, the treatment given is a complete one. Using the same method, Soviet venereologists developed the so-called "ring" method of treating syphilis cases, which is specially adapted to rural conditions. Patients who live in various villages are seen by a venereologist once a week as provided for by a special schedule, and are treated in their own villages.

Every 2 or 3 years the methods of treatment are reviewed at all-Union conferences. For example, Mapharsen and so-called "accelerated" methods of treating syphilis were widely used in the U.S.S.R. between 1944 and 1948. However, the investigation of the immediate and remote results of treatment showed that relapses including neurosyphilis occurred in 10 to 17 per cent. of the cases. This led Soviet syphilologists to give up these preparations and methods entirely.

Wide use is made of the system of post-graduate medical courses. Besides the knowledge doctors 
receive in the medical institutions, they specialize later in the field of dermatology and venereology. To attain this goal they spend 3 or 4 months in one of the postgraduate institutes or other dermatovenereological institutions. After a doctor has worked for 5 years in his field, he has the right to join refresher courses for 4 months. The opportunity for specialization and betterment of qualifications is given free of charge. Salaries are paid during the whole period of training and travelling expenses are defrayed. This system provides for the continuous improvement of the doctor's knowledge, which is reflected in the great improvement of the health services.

As a result of the work done, the incidence of syphilis showed a sharp drop by 1941. In 1924 in Moscow 7.4 primary syphilis cases were registered per 10,000 persons; in 1939 there were only 0.87 cases per 10,000 , i.e. the incidence of primary syphilis had decreased nine-fold. In 1913 in Leningrad 25.6 new syphilis cases (both primary and secondary) were registered per 10,000 persons, and in 1940 there were only 2.4 cases. Throughout 1940 only two to ten new cases were registered in such big cities as Smolensk, Molotov, Ryazan, Ufa, Tambov, Yaroslavl, Ivanovo, Orel, Saratov, Poltava, and Irkutsk.

World War II retarded the decrease of the incidence of syphilis in the U.S.S.R. Venereological institutions were destroyed in the territories occupied by the Germans, and the treatment of syphilis cases was almost completely stopped, though the incidence of the disease was especially high in the occupied parts of the country. For example, at the beginning of the war the number of syphilis cases in Kharkov was 0.9 per 10,000 persons, and it rose to 34 per 10,000 during the occupation.

After the war was over the incidence of syphilis began to decrease steadily. Taking 100 to indicate the highest post-war incidence, in 1951 primary syphilis cases fell to $12 \cdot 8$ and secondary syphilis cases to $9 \cdot 6$. The incidence of active forms of syphilis in the U.S.S.R. showed a 19.4-fold decrease by 1955 , compared with 1945 . In the Western Ukraine, taking the number of active syphilis cases as 100 in 1945 , by 1952 their number had decreased 82 -fold in the Lvov Region and 50-fold in the Drogobych and Ternopol regions. At present (1955) the number of new syphilis cases is so small that in most regions not a single case was registered during the whole year. The number of progressive paralysis and tabes dorsalis cases decreased 150 -fold approximately compared with the prerevolution figure (Table).

\section{TABLE}

INCIDENCE OF SYPHILIS IN WESTERN UKRAINE, 1945-52*

\begin{tabular}{c|c|c|c|c}
\hline Year & Lvov & Drogobych & Ternopol & Tchernowitz \\
\hline 1945 & 100 & 100 & 100 & 100 \\
1948 & 37.9 & 89 & 88 & $51 \cdot 5$ \\
1952 & 1.22 & 2 & 2 & 0.53 \\
\hline
\end{tabular}

* From Vestnik Venereologii i Dermatologii, 1954, 6, 42.

In view of the successes achieved, the health protection organizations of the U.S.S.R. are now tackling the task of completely eliminating the disease within the next few years. At present all cases of early congenital, recidive, and tertiary syphilis are regarded as failures of medical work and are discussed at conferences of medical workers. The assessment of results of the work done in the field of syphilis control provides syphilologists with the necessary data to be applied in the planning of work, developing of correct forms and methods, etc.

The results achieved in this field cannot be ascribed to the functioning of venereological institutions alone. Of decisive importance were the improvement of living conditions and the well-being of the population, elimination of unemployment and prostitution, steady growth of Soviet culture, a good system of mother and child welfare, and large allocations trom state funds for the needs of health protection and education. 